\title{
About an optimal visiting problem
}

\author{
Fabio Bagagiolo* and Michela Benetton
}

\begin{abstract}
In this paper we are concerned with the optimal control problem consisting in minimizing the time for reaching (visiting) a fixed number of target sets, in particular more than one target. Such a problem is of course reminiscent of the famous "Traveling Salesman Problem" and brings all its computational difficulties. Our aim is to apply the dynamic programming technique in order to characterize the value function of the problem as the unique viscosity solution of a suitable Hamilton-Jacobi equation. We introduce some "external" variables, one per target, which keep in memory whether the corresponding target is already visited or not, and we transform the visiting problem in a suitable Mayer problem. This fact allows us to overcome the lacking of the Dynamic Programming Principle for the originary problem. The external variables evolve with a hysteresis law and the Hamilton-Jacobi equation turns out to be discontinuous.
\end{abstract}

Keywords: visiting problem, minimum time, hysteresis, dynamic programming, discontinuous Hamilton-Jacobi equations, viscosity solutions, traveling salesman problem.

\section{Introduction}

By "optimal visiting problem" in this paper we mean the following optimal control problem. Let us consider the controlled dynamical system in $\mathbb{R}^{n}$

$$
\left\{\begin{array}{l}
y^{\prime}(t)=f(y(t), \alpha(t)) \quad t>0 \\
y(0)=x
\end{array}\right.
$$

*Dipartimento di Matematica, Unversità di Trento, Via Sommarive 14, 38050 Trento, Italy, bagagiol@science.unitn.it 
where $\alpha:[0,+\infty[\rightarrow A$ is the measurable control and the dynamic $f$ is suitably regular, and consider $m$ targets given by $m$ closed disjoint sets

$$
\mathcal{T}_{j} \subset \mathbb{R}^{n} j=1, \ldots, m .
$$

The optimal visiting problem consists in minimizing, above all measurable controls, the necessary time for the trajectory $y$ of (1.1) for "touching" all the targets. For every control $\alpha$ we may define the visiting time, starting from a point $x \in \mathbb{R}^{n}$,

$$
t_{x}(\alpha)=\inf \left\{t \geq 0 \mid \exists t_{1}, \ldots, t_{m} \in[0, t], y\left(t_{j}\right) \in \mathcal{T}_{j} \forall j=1, \ldots, m\right\}
$$

In particular, note that we have not fixed any order for the visit of the targets. We then consider the "minimum visiting" function

$$
T(x)=\inf _{\alpha} t_{x}(\alpha) .
$$

The goal of the present paper is to characterize the minimum visiting function as the unique viscosity solution of a suitable HamiltonJacobi equation. To do that we of course need the validity of the Dynamic Programming Principle. Unfortunately, this is not the case: the presence of more than one target makes immediately fail the Principle. Actually, we may still in some sense recover the Dynamic Programming Principle but paying the price that we have to decompose the problem in a great number of sub-problems (exponentially increasing with $m$, the number of targets), and so, whenever we would like to calculate the minimum visiting function we are forced to solve a great number of sub-equations. This last fact is of course similar to what happens in the famous Traveling Salesman Problem, to which our problem is certainly linked. Indeed that problem consists in searching a path which minimizes the total length of the route a traveling salesman has to follow in order to reach a certain number of towns. This can be certainly viewed as an optimal visiting problem as above, where the dynamics $f$ has constant norm equal to 1 , and so minimizing the time is equivalent to minimizing the length among all admissible paths.

The aim of this paper is to overcome such a decomposition in subproblems, and write a unique Hamilton-Jacobi equation able to characterize the optimal visiting function as its unique viscosity solution. To get such a goal we add to the problem some "external variables" $w_{1}(\cdot), \ldots, w_{m}(\cdot)$, one per target, such that $w_{j}(t) \geq 0$ and $w_{j}(t)=0$ if 
and only if $y(\tau) \in \mathcal{T}_{j}$ for some $0 \leq \tau \leq t$. The evolutions of the variables $w_{j}$, subject to the evolution of $y$, are represented by a so-called "hysteresis law" which presents a particular kind of memory, and satisfies a suitable "semigroup property" which allows us to completely recover the Dynamic Programming Principle. In particular, "memory" also stands for the fact that, whenever $w_{j}(t)=0$ (which means that the $j$-th target $\mathcal{T}_{j}$ is already reached), then $w_{j}(s)=0$ for all $s \geq t$. We then transform the optimal visiting problem in a suitable Mayer problem. Namely, we define the function

$$
\Psi: \mathbb{R}^{n} \times \mathbb{R}^{m} \rightarrow \mathbb{R}, \quad\left(x, w_{1}, \ldots, w_{m}\right) \mapsto w_{1}+\cdots+w_{m},
$$

and hence, still referring to the controlled system (1.1) and denoting by $w^{0}$ the initial state of the variable $w=\left(w_{1}, \ldots, w_{m}\right)$ whose evolution is $w(\cdot)$, we consider the optimal control problem given by

$$
V\left(t, x, w^{0}\right)=\inf _{\alpha} \Psi(y(t), w(t)) .
$$

It is evident that, for any initial datum $w^{0}$ with $w_{j}^{0}>0$ for all $j=$ $1, \ldots, m$, the optimal visiting function $T(1.2)$ satisfies

$$
T(x)=\inf \left\{t \geq 0 \mid V\left(t, x, w^{0}\right)=0\right\},
$$

and hence, if we know the value function $V$, we can reconstruct the optimal visiting function $T$.

We then perform the study of the value function $V$ (1.3). After proving its continuity in a suitable space $\bar{\Omega} \subset \mathbb{R}^{n} \times \mathbb{R}^{m}$, we prove that it is the unique viscosity solution of the following Cauchy problem for a discontinuous Hamilton-Jacobi equation

$$
\left\{\begin{array}{l}
\left.V_{t}(y, w, t)+H\left(y, w, D_{y} V(y, w, t), D_{w} V(y, w, t)\right)=0 \text { in } \bar{\Omega} \times\right] 0,+\infty[ \\
V(y, w, 0)=\Psi(y, w)
\end{array}\right.
$$

with

$H(y, w, p, q):=\sup _{a \in A}\left\{-f(y, a) \cdot p+\sum_{j=1}^{m} q_{j} \chi\left(g_{j}(y), w_{j}\right)\left(D g_{j}(y) \cdot f(y, a)\right)^{-}\right\}$,

where $V_{t}, D_{y} V, D_{w} V$ indicate partial derivatives, $\chi$ denotes the characteristic function of the bisector of the first quadrant in $g_{j}(y)-w_{j}$ plane, where $g_{j}$ are suitable functions, and finally $(\cdot)^{-}$stays for the negative part. 
The price we pay for having added the variable $w$ (and hence for the possibility of writing a unique equation) is given by the fact that the equation in (1.4) is discontinuous. To prove the uniqueness result we transform it in a continuous equation with suitable boundary conditions of Neumann type.

For the general viscosity solutions theory and for applications to Hamilton-Jacobi equations, we refer the reader to the book by Bardi and Capuzzo Dolcetta [3], as well as to the seminal papers CrandallLions [6] and Crandall-Evans-Lions [7]. For what concerns mathematical hysteresis models, and in particular the so-called hysteresis operators, we refer to the book by Visintin [14]. An optimal control problem with hysteresis evolution, similar to the present one, was studied in Bagagiolo [1]. In that article, however, (besides an infinite dimensional problem) the state variable is only one-dimensional, only one hysteresis operator is applied (instead of $m$ operators as in the present case), and the optimal control is of infinite horizon type, i.e. without explicit dependence on time, as instead the Mayer problem is. In the last decade, control and optimal control problems for ODEs with hysteresis have been the subject of an increasing number of articles, see for instance Gudovich-Quincampoix [9], Ilchmann-Logemann-Ryan [10], Bagagiolo [2] and the references therein. Finally, the literature concerning the Traveling Salesman Problem is of course very huge, and also several versions of suitable dynamic programming algorithms are intensively studied via optimization techniques. However, we do not report here anything of that but a little paper by R. Bellman [4], where a possible dynamic programming approach to the problem is proposed. In that paper, in order to make the Dynamic Programming Principle hold, some external variables are indeed inserted, but they do not evolve, they are stationary, and no Hamilton-Jacobi (Bellman) equation is written down, only some calculation of the numbers of sub-problems is performed. Indeed, it seems that our paper, and this is one of its novelty, is the first one to write and to study a partial differential equation of Hamilton-Jacobi type, linked to problems of Traveling-Salesman nature.

The article is organized as it follows. In Chapter 2 we expose all the reasoning that lead us to formulate the optimal visiting problem as a Mayer problem for a controlled evolution with hysteresis. In Chapter 3 we recall some basic facts and some properties concerning the particular hysteresis operator we are going to consider, and concerning viscosity solutions for discontinuous Hamilton-Jacobi equations, 
as well as for the Neumann boundary problem. In Chapter 4 we rigorously formulate the Mayer problem, we prove the continuity of the value function, and we derive the discontinuous Hamilton-Jacobi equation satisfied by the value function in the viscosity sense. In Chapter 5 we transform the discontinuous equation in a continuous one with boundary conditions in the Neumann type, and we prove the uniqueness result. Finally in Chapter 6 we give some remarks and extensions about other possible formulations and/or approximations of the optimal visiting problem.

\section{Reasoning about the problem and the model}

In this section we analyze the following facts: the general non validity of the Dynamic Programming Principle for the case with $m>1$ targets; a first almost natural solution to that problem: the decomposition in many sub-problems; the introduction of the external variables; a first possible new formulation of the problem, but the non continuity of the value function.

In a following section we are going to describe the Mayer problem which, in some sense, bypasses all those problems.

\subsection{Lacking of dynamic programming}

As it is well known, the Dynamic Programming Principle can be expressed informally by: pieces of optimal trajectories are optimal. Namely, fixing any point along an optimal trajectory, the remaining piece of trajectory results to be optimal for that fixed point. In order to solve our optimal visiting problem, every target set $\mathcal{T}_{j}$ has to be reached. Consequently, when there are more than one target, the Dynamic Programming Principle does not hold: an optimal trajectory $y(\cdot)$ is not necessary optimal for every point on it. Indeed, If we consider for example an instant $t>0$ such that $y(t)$ has already reached one of the targets, let us say $\mathcal{T}_{j}$, then the remaining part of trajectory $y(\cdot)$ with initial state $y(t)$ has no reason to be optimal for $y(t)$ : it probably even does not touch the target $\mathcal{T}_{j}$ anymore. 


\subsection{Decomposition into sub-problems}

We can think to solve our optimal visiting problem by minimizing the time spent by the trajectory, with initial state $x \in \mathbb{R}^{n}$, for reaching one of the targets $\mathcal{T}_{k}, k \in\{1, \ldots, m\}$, plus a cost $T_{k}$ defined on $\partial \mathcal{T}_{k}$. Here $T_{k}$ on $\partial \mathcal{T}_{k}$ is given by the minimum time to visit all the other targets $\mathcal{T}_{j} j \in\{1, \ldots, m\} \backslash\{k\}$. In this way, the optimal visiting problem is similar to a minimal time problem with target $\bigcup \mathcal{T}_{j}$ with a "reachingcost" given by the functions $T_{j}$ on the boundaries $\partial \mathcal{T}_{j}$. In particular, with suitable assumptions, the Dynamic Programming Principle holds and the minimal time function $T$ can be characterized as the unique continuous and lower bounded solution of

$$
\left\{\begin{array}{lc}
\sup _{a}\{-f(x, a) \cdot D T(x)\}=1 & \text { in } \mathcal{R} \backslash \bigcup_{j=1}^{m} \mathcal{T}_{j} \\
T \rightarrow+\infty & x \rightarrow \partial \mathcal{R} \\
T(x)=T_{j}(x) & \forall x \in \partial \mathcal{T}_{j} \forall j=1, \ldots, m
\end{array}\right.
$$

where $\mathcal{R}$ is the set of controllable starting points. So, before analyzing such a problem, we have to calculate all the functions $T_{j}$ on $\partial \mathcal{T}_{j}$ for $j=1, \ldots, m$, which means that we must solve $m$ optimal visiting problems with $m-1$ targets. Each one of such problems leads us to face up other sub-problems with $m-2$ targets, and so on. In conclusion, with this procedure, we have to solve all the possible optimal visiting sub-problems with a number of targets less than $m$.

\subsection{Introduction of the external variables}

To overcome the lacking of the Dynamic Programming Principle in section 2.1, and the decomposition in several sub-problems in section 2.2 , we add new variables, one per target, that register in every instant $t>0$ whether a target has been already visited or not. Such variables may be initially thought as

$$
w_{j}(t)=\min _{\tau \in[0, t]}\left\{\operatorname{dist}\left(y(\tau), \mathcal{T}_{j}\right), w_{j}^{0}\right\}=: S P\left[\operatorname{dist}\left(y(\cdot) ; \mathcal{T}_{j}\right), w_{j}^{0}\right](t),
$$

where $w_{j}^{0}$ is a suitable initial state and $S P$ stays for the "Semiplay operator of hysteresis", which will be introduced later on, and is suitable to represent such an evolution.

The new state of the problem is then $\left(x, w_{1}, \ldots, w_{m}\right) \in \mathbb{R}^{n} \times \mathbb{R}^{m}$. Now, even supposing that the targets are convex, the distance function 
from one of them, let us say $\mathcal{T}_{k}$, is $\mathcal{C}^{1}$ only in $\mathbb{R}^{n} \backslash \mathcal{T}_{k}$, hence it may be convenient to add a state constraint to enforce the trajectory $y(\cdot)$ stays out of the targets (if the targets are planets to be reached by a spacecraft, this is obviously a natural assumption). This is certainly possible to be studied (see Remark 6.1), however here we suppose that we can go through the targets, (they can be thought as cities to be reached by the traveling salesman). Therefore instead of the distance function we consider some suitable scalar functions $g_{j}: \mathbb{R}^{n} \rightarrow \mathbb{R}$ such that

$$
g_{j} \in \mathcal{C}^{\infty}, \quad g_{j} \geq 0 \text { and } g_{j}(x)=0 \Leftrightarrow x \in \mathcal{T}_{j},
$$

and hence the evolution of the new variables is given by

$$
w_{j}(t)=S P\left[\left(g_{j} \circ y\right)(\cdot), w_{j}^{0}\right](t) .
$$

\subsection{A natural model with non continuous value function}

With the external variables introduced above, the controlled system is

$$
\begin{cases}y^{\prime}(t)=f(y(t), \alpha(t)) & t>0 \\ w_{j}(t)=S P\left[g_{j} \circ y, w_{j}^{0}\right](t) & \forall j=1, \ldots, m \\ y(0)=x & x \in \mathbb{R}^{n} \\ w_{j}^{0} \leq g_{j}(x) & \forall j=1, \ldots, m\end{cases}
$$

where $S P$ is the Semiplay hysteresis operator with continuous output $w_{j}(t)$. Every initial state $(x, w)=\left(x, w_{1}^{0} \ldots, w_{m}^{0}\right)$ belongs to the closure $\bar{\Omega}$ of the following set

$$
\Omega:=\left\{(x, w) \in \mathbb{R}^{n} \times \mathbb{R}^{m}: 0<w_{j}<g_{j}(x) \quad \forall j=1, \ldots, m\right\} .
$$

Coherently with the definitions given till now, by the choice of functions $g_{j}$ and the properties of Semiplay operator, we may interpret the optimal visiting problem as a minimum time problem in $\mathbb{R}^{n} \times \mathbb{R}^{m}$, subject to (2.5), with the (unique) target

$$
\mathcal{T}:=\mathbb{R}^{n} \times\left\{w \in \mathbb{R}^{m}: w_{j}=0 \quad \forall j=1, \ldots, m\right\} .
$$

The trajectory $\left(y_{\left(x, w^{0}\right)}(\cdot ; \alpha), w_{\left(x, w^{0}\right)}(\cdot ; \alpha)\right)$ with initial state $\left(x, w^{0}\right) \in$ $\bar{\Omega} \backslash \mathcal{T}$ and control $\alpha$ has reaching time

$$
t_{\left(x, w^{0}\right)}(\alpha):=\inf \left\{t>0:\left(w_{\left(x, w^{0}\right)}(t ; \alpha)\right)_{j}=0 \quad j=1, \ldots, m\right\},
$$


where $(v)_{j}$ stays for the $j$-th component of the vector $v$. The minimum time function is then

$$
T\left(x, w^{0}\right):=\inf _{\alpha}\left\{t_{\left(x, w^{0}\right)}(\alpha)\right\} .
$$

Unfortunately, the function $T$ (2.6) is not continuous at points with, for at least one $j=1, \ldots, m, w_{j}=0$ and $g_{j}(x)>0$,. Indeed, for such initial states $(x, w) \in \bar{\Omega}$, the trajectory will ignore the target $\mathcal{T}_{j}$ (since it is, in some sense, already reached). However, around to $(x, w)$, a point $(y, z)$ could be such that $z_{j}=\varepsilon>0$. So the trajectory with initial state $(y, z)$ must consider $\mathcal{T}_{j}$ and reach it in a time $t>0$ that does not tend to zero for $\varepsilon \rightarrow 0$. Then $T(y, z)$ does not converge to $T(x, w)$.

\section{Some tools and results}

In this section we define Semiplay operator of hysteresis and we prove its main properties, which we shall use in the following sections.

Next we give the necessary definitions to introduce the concept of viscosity solution for discontinuous Hamilton-Jacobi equation and for a Neumann problem.

\subsection{The Semiplay operator of hysteresis}

Hysteresis is a particular memory-type relationship (the so-called rate independent one) between a continuous time-dependent input $u$ and a time-dependent output $w$. It can be often suitably described by the concept of hysteresis operator, that is a suitable operators between some functional spaces (see Visintin [14]). Here, for our purposes, we introduce a particular example of such operators, and we call it the Semiplay operator of hysteresis.

For every $T>0$, let us consider the following sets

$$
\begin{aligned}
& \Sigma:=\left\{(\xi, w) \in \mathbb{R}^{2}: w<\xi\right\}, \\
& \mathcal{D}=\left\{\left(u, w^{0}\right) \in \mathcal{C}^{0}([0, T]) \times \mathbb{R}:\left(u(0), w^{0}\right) \in \bar{\Sigma}\right\}
\end{aligned}
$$

The Semiplay operator $S P: \mathcal{D} \rightarrow \mathcal{C}^{0}([0, T])$, with output $w(\cdot):=$ $S P\left[u ; w^{0}\right](\cdot)$, is defined by the following theorem. Let $\partial I_{[0,+\infty[}$ be the 
subdifferential of the indicator function $I_{[0,+\infty[}$ of the set $[0,+\infty[\subset \mathbb{R}$ $\left(I_{[0,+\infty[}(x)=0\right.$ if $x \geq 0, I_{[0,+\infty[}(x)=+\infty$ if $\left.x<0\right)$ :

$$
\partial I_{[0,+\infty[}(x)= \begin{cases}\{0\} & \text { if } x \in] 0,+\infty[ \\ ]-\infty, 0] & \text { if } x=0\end{cases}
$$

Theorem 3.1. Given an input $u \in W^{1,1}(0, T)$ and an initial state $w^{0} \in \mathbb{R}$ such that $\left(u(0), w_{0}\right) \in \bar{\Sigma}$, the output $w(\cdot)=S P\left[u ; w^{0}\right](\cdot)$ is characterized as the unique solution $w \in W^{1,1}(0, T)$ of the following differential inclusion:

$$
\left\{\begin{array}{l}
w^{\prime}(t) \in \partial I_{[0,+\infty}[(u(t)-w(t)) \quad \text { for a.e. } t \in] 0, T[ \\
w(0)=w^{0}
\end{array}\right.
$$

Moreover: the Semiplay operator is Lipschitz continuous with Lipschitz constant 1 , that is $\forall(u, w),(v, z) \in \mathcal{D}$

$$
\|S P[u, w]-S P[v, z]\|_{\mathcal{C}^{0}([0, T])} \leq\|u-v\|_{\mathcal{C}^{0}([0, T])}+|w-z| ;
$$

the Semiplay operator satisfies a semigroup property, that is for every $\left(u, w^{0}\right) \in \mathcal{D}$ and for every $t, \tau \in[0, T]$ with $t+\tau \leq T$, let $w(t):=$ $S P\left[u, w^{0}\right](t)$ we have

$$
S P\left[u, w^{0}\right](t+\tau)=S P[u(t+\cdot), w(t)](\tau) .
$$

Proof. The Semiplay is a particular case of the so-called Play operator of hysteresis. Fixed $\rho>0$ and defined the strip $\Sigma_{\rho}:=\{(\xi, w) \in$ $\left.\mathbb{R}^{2}: \xi-\rho<w<\xi\right\}$, the output of the Play operator

$$
P_{\rho}:\left\{\left(u, w^{0}\right) \in \mathcal{C}^{0}([0, T]) \times \mathbb{R}:\left(u(0), w^{0}\right) \in \bar{\Sigma}_{\rho}\right\} \rightarrow \mathcal{C}^{0}([0, T])
$$

is characterized as the unique solution $w \in W^{1,1}(0, T)$ of

$$
\left\{\begin{array}{l}
w^{\prime}(t) \in \partial I_{[-\rho, \rho]}(u(t)-w(t)) \quad \text { a.e. } t \in(0, T) \\
w(0)=w^{0} .
\end{array}\right.
$$

This is indeed a good definition (i.e. there exists one and only one solution), as it is proved in Visintin [14], where it is also proved that the Play operator $P_{\rho}$ is Lipschitz continuous with constant 1 and satisfies the semigroup property. Given an input $u \in W^{1,1}(0, T)$ and defined $a=\max \{|u(t)-u(0)| \mid t \in[0, T]\}$, it is not difficult to see that $S P\left[u, w^{0}\right]=P_{2 a}\left[u, w^{0}\right]$, from which the conclusion follows.

Note that the name "Semiplay" just means that the strip $\Sigma$ is bounded from only one side, and not from both sides as for the Play operator. 

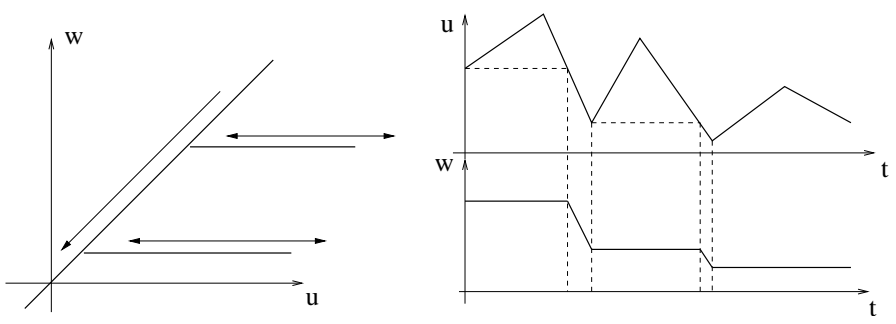

Figure 1: SemiPlay hysteresis operator

Remark 3.2. Figure 1 describes the behavior of the output $w$ of the Semiplay operator: on the left it is depicted a sort of phase portrait of the hysteretic relationship in the input-output plane, $u-w$; on the right the graph of an input $u$ and of the corresponding output $w$, with the same initial state $u(0)=w^{0}$, are reported.

Theorem 3.1 expresses what is represented in Figure 1, that is:

- $w^{\prime}(t)=0$ if $u(t)-w(t)>0$, i.e. $w(t)$ is constant if $u(t)>w(t)$;

- $w^{\prime}(t) \leq 0$ if $u(t)-w(t)=0$, i.e $w(t)$ is nonincreasing if $u(t)=$ $w(t)$.

As a consequence of the same theorem, in particular of the definition of the subdifferential, we have also that $u(t) \geq w(t)$ for every $t \in] 0, T[$, that is $(u(t), w(t)) \in \Sigma$ for all $t$; moreover we also have $u^{\prime}(t)=w^{\prime}(t)$ whenever $w(t)=u(t)$ and $u^{\prime}(t) \leq 0$ (for a.e. $t$ ).

Finally, let us note that if we take as input u the distance function as in section 2.3, then the output $w$ has exactly the desired behavior, that is it represents the minimal distance from the target reached by $y$ during its evolution. We may similarly interpret the behavior of $w$ when the input is not more the distance function from the target $\mathcal{T}_{j}$ but instead the function $g_{j} \circ y$. In such a case the output $w=S P\left[g_{j} \circ y, w^{0}\right]$ is exactly storing the minimal value reached by $g_{j} \circ y$ and so it is null if and only if $y$ has already reached the target.

The following results characterizes the output of the Semiplay operator as a solution of a discontinuous differential equation. Let us denote by $\chi(\cdot, \cdot)$ the characteristic function of the straight line in the $(\xi, w)$-plane of equation $w=\xi$ (i.e. $\quad \chi(\xi, w)=1$ if $w=\xi$, $\chi(\xi, w)=0$ otherwise). For $a \in \mathbb{R}$ we indicate its negative part by $a^{-}:=\max \{-a, 0\}$. 
Theorem 3.3. For every $u \in W^{1,1}(0, T)$ and for every admissible initial state $w^{0} \in \mathbb{R}$, the output $w$ of the Semiplay operator satisfies the following discontinuous differential equation:

$$
\left.w^{\prime}(t)=-\chi(u(t), w(t))\left(u^{\prime}(t)\right)^{-} \quad \text { for a.e. } t \in\right] 0, T[.
$$

Proof. Let $t \in(0, T)$ be such that $w^{\prime}(t)$ and $u^{\prime}(t)$ exist. If $u(t)-$ $w(t)>0$, we get the thesis as a consequence of sign's permanence theorem and Theorem 3.1. Whereas if $u(t)=w(t)$, we can prove the following relations (the second one along a suitable sequence $h_{n} \rightarrow 0$ ):

$$
\begin{aligned}
& u(t) \leq 0 ; \\
& w^{\prime}(t)=\lim _{n \rightarrow+\infty} \frac{w\left(t+h_{n}\right)-w(t)}{h_{n}}=\lim _{n \rightarrow+\infty} \frac{u\left(t+h_{n}\right)-u(t)}{h_{n}}=u^{\prime}(t) .
\end{aligned}
$$

through which we conclude (see also Bagagiolo [1] for explicit calculations in the case of the Play operator).

\subsection{Viscosity solutions for discontinuous H-J equations}

Let $\Omega \subseteq \mathbb{R}^{n}$ be a set, $\left.\left.T \in\right] 0,+\infty\right], H: \Omega \times \mathbb{R}^{n} \rightarrow \mathbb{R}$ a function, and consider the Hamilton-Jacobi equation:

$$
\left.u_{t}(x, t)+H\left(x, D_{x} u(x, t)\right)=0 \quad \text { in } \Omega \times\right] 0, T[,
$$

where $u_{t}$ and $D_{x}$ respectively stay for the time derivative and for the spatial gradient of the unknown function $u: \Omega \times] 0, T[\rightarrow \mathbb{R}$. In order to handle possible discontinuities of the Hamiltonian $H$, following Ishii [11], we introduce the following variant for the classical definition of viscosity solutions, which involves the lower semicontinuous and upper semicontinuous envelopes of $H$ :

$$
H_{*}(x, p)=\liminf _{\left(y, p^{\prime}\right) \rightarrow(x, p)} H\left(y, p^{\prime}\right), \quad H^{*}(x, p)=\limsup _{\left(y, p^{\prime}\right) \rightarrow(x, p)} H\left(y, p^{\prime}\right) .
$$

Definition 3.4. A function $u \in \mathcal{C}(\Omega \times] 0, T[)$ is said to be a

i) "viscosity subsolution" of (3.11) if, for every $\varphi \in \mathcal{C}^{1}(\Omega \times] 0, T[)$ and $\left.\left(x_{0}, t_{0}\right) \in \Omega \times\right] 0, T[$ local maximum point for $u-\varphi$ : 


$$
\varphi_{t}\left(x_{0}, t_{0}\right)+H_{*}\left(x_{0}, D_{x} \varphi\left(x_{0}, t_{0}\right)\right) \leq 0 .
$$

ii) "viscosity supersolution" of (3.11) if, for every $\varphi \in \mathcal{C}^{1}(\Omega \times] 0, T[)$ and $\left.\left(x_{0}, t_{0}\right) \in \Omega \times\right] 0, T[$ local minimum point for $u-\varphi$ :

$$
\varphi_{t}\left(x_{0}, t_{0}\right)+H^{*}\left(x_{0}, D_{x} \varphi\left(x_{0}, t_{0}\right)\right) \geq 0 .
$$

iii) "viscosity solution" of (3.11) if it is simultaneously a viscoscosity sub- and supersolution.

The following lemma asserts that, if $T$ is finite, we can push the property of being solution till to the upper closure ]0,T].

Lemma 3.5. Referring to Definition 3.4, if $u \in \mathcal{C}(\Omega \times] 0, T])$ is a viscosity subsolution (respectively supersolution) of (3.11), then it is also a subsolution (respectively a superoslution) in $\Omega \times] 0, T]$, that is

$$
\varphi_{t}\left(x_{0}, t_{0}\right)+H_{*}\left(x_{0}, D_{x} u\left(x_{0}, t_{0}\right)\right) \leq 0
$$

(respectively $\geq 0$ with $H^{*}$ ) for every local maximum (respectively minimum) point $\left(x_{0}, t_{0}\right)$ of $u-\varphi$ in $\left.\left.\Omega \times\right] 0, T\right]$, where $\left.\left.\varphi \in \mathcal{C}^{1}(\Omega \times] 0, T\right]\right)$.

Proof. The main ingredient of the proof is the monotonicity of the equation with respect to the time derivative. In Bardi-Capuzzo Dolcetta [3], it can be found the proof for continuous Hamiltonians $H$, however continuity is not necessary: the semicontinuity of $H_{*}$ and $H^{*}$ is enough.

\subsection{Neumann problem}

In this section we suppose $\Omega$ open, $H$ continuous, we consider a regular set $\Gamma \subseteq \partial \Omega$, and a continuous outward vector $\nu(\cdot)$ on $\Gamma$.

A viscosity subsolution (respectively supersolution) of the boundary value problem of Neumann type in the viscosity sense

$$
\left\{\begin{array}{lc}
u_{t}(x, t)+H\left(x, D_{x}(x, t)\right)=0 & \text { in }(\bar{\Omega} \backslash \Gamma) \times] 0, T[ \\
D_{x}(x, t) \cdot \nu(x)=0 & \text { on } \Gamma \times] 0, T[
\end{array}\right.
$$

is a continuous function $u: \bar{\Omega} \times] 0, T[\rightarrow \mathbb{R}$ such that, for every test function $\varphi \in C^{1}(\bar{\Omega} \times] 0, T\left[\right.$ ), and for every $\left.\left(x_{0}, t_{0}\right) \in \bar{\Omega} \times\right] 0, T[$ maximum (respectively minimum) point for $u-\varphi$ in $\bar{\Omega} \times] 0, T[$ 


$$
\left\{\begin{array}{c}
\varphi_{t}\left(x_{0}, t_{0}\right)+H\left(x_{0}, D_{x} \varphi\left(x_{0}, t_{0}\right)\right) \leq 0, \\
\left(\operatorname{resp.} \varphi_{t}\left(x_{0}, t_{0}\right)+H\left(x_{0}, D_{x} \varphi\left(x_{0}, t_{0}\right)\right) \geq 0\right) \\
\left.\quad \text { if }\left(x_{0}, t_{0}\right) \in(\bar{\Omega} \backslash \Gamma) \times\right] 0, T[, \\
\min \left\{D_{x} \varphi\left(x_{0}, t_{0}\right) \cdot \nu\left(x_{0}\right), \varphi_{t}\left(x_{0}, t_{0}\right)+H\left(x_{0}, D_{x} \varphi\left(x_{0}, t_{0}\right)\right)\right\} \leq 0, \\
\left(\max \left\{D_{x} \varphi\left(x_{0}, t_{0}\right) \cdot \nu\left(x_{0}\right), \varphi_{t}\left(x_{0}, t_{0}\right)+H\left(x_{0}, D_{x} \varphi\left(x_{0}, t_{0}\right)\right)\right\} \geq 0\right) \\
\left.\quad \text { if }\left(x_{0}, t_{0}\right) \in \Gamma \times\right] 0, T[,
\end{array}\right.
$$

The first notion of such a boundary condition, can be found in Lions [12]. See also Day [8] for the case of reflected dynamics, which is linked to our hysteresis problem (also see Bagagiolo [1]).

\section{Study of the Mayer problem}

In this section the Mayer problem is rigorously formulated and studied with Dynamic Programming technique.

\subsection{The Mayer problem}

Referring to Section 2.4 for motivation, given $m$ scalar functions $g_{j}$ : $\mathbb{R}^{n} \rightarrow \mathbb{R}$ such that

$$
g_{j} \geq 0, g_{j} \in \mathcal{C}^{2} \text { and Lipschitz continuous, }
$$

we consider the dynamic system

$$
\begin{cases}y^{\prime}(t)=f(y(t), \alpha(t)) & t>0 \\ w_{j}(t)=S P\left[g_{j} \circ y, w_{j}^{0}\right](t) & \forall j=1, \ldots, m \\ y(0)=x & x \in \mathbb{R}^{n} \\ w_{j}^{0} \leq g_{j}(x) & \forall j=1, \ldots, m\end{cases}
$$

where $S P$ is the Semiplay operator of hysteresis as in Section 3.1. The measurable control $\alpha:[0,+\infty[\rightarrow A$ takes value in the compact set $A \subset \mathbb{R}^{k}$ for some $k \in \mathbb{N}$ and the set of all measurable controls is $\mathcal{A}$. The dynamics $f: \mathbb{R}^{n} \times A \rightarrow \mathbb{R}^{n}$ is continuous and satisfies:

$$
\begin{aligned}
& \exists M, L>0 \text { such that }|f(x, a)| \leq M \quad \forall a \in A, \forall x \in \mathbb{R}^{n} ; \\
& \quad|f(x, a)-f(z, a)| \leq L|x-z| \quad \forall a \in A, \forall x, z \in \mathbb{R}^{n} .
\end{aligned}
$$

A solution (trajectory) of (4.14) is a continuous function $(y, w)$ : $\left[0,+\infty\left[\rightarrow \mathbb{R}^{n} \times \mathbb{R}^{m}\right.\right.$ such that $w_{j}(t)=S P\left[g_{j} \circ y, w_{j}^{0}\right](t)$ for all $t \geq 0$ and 


$$
y(t)=x+\int_{0}^{t} f(y(s), \alpha(s)) d s, \quad \forall t \geq 0 .
$$

Proposition 4.1. Let us suppose that (4.13) and (4.15) hold. Then for every initial state $\left(x, w^{0}\right) \in \mathbb{R}^{n} \times \mathbb{R}^{m}$, such that $w_{j}^{0} \leq g_{j}(x)$ for all $j=1, \ldots, m$, and for every control $\alpha \in \mathcal{A}$, there exists a unique solution of the system (4.14), which we denote by $\left(y_{\left(x, w^{0}\right)}(\cdot ; \alpha), w_{\left(x, w^{0}\right)}(\cdot ; \alpha)\right)$.

Proof. The proof follows from standard techniques for ordinary differential equations, in particular from the boundedness and Lipschitz continuity of $f$ (4.15), the Lipschitz continuity if $g_{j}$ (4.13), and from Lipschitz continuity of $S P$ operator (3.8).

In order to constrain, for simplicity, the trajectories inside a bounded set, we suppose that there exists $\mathcal{B} \subseteq \mathbb{R}^{n}$ open, regular and bounded such that, denoted by $\nu$ the outward normal vector:

$$
f(x, a) \cdot \nu(x)<0 \quad \forall x \in \partial \mathcal{B}, \forall a \in A .
$$

Hence, as set of admissible initial states, we consider the closure, $\bar{\Omega}$, of the open bounded set:

$$
\Omega:=\left\{(x, w) \in \mathcal{B} \times \mathbb{R}^{m}: 0<w_{j}<g_{j}(x) \quad \forall j=1, \ldots, m\right\} .
$$

Remark 4.2 Note that by the hypothesis (4.16), by the non-negativity of the function $g_{j}$, and by the properties of the $S P$ operator, the bounded set $\bar{\Omega} \subset \mathbb{R}^{n} \times \mathbb{R}^{m}$ is invariant for the trajectories of (4.14), that is, for every measurable control and for every initial state in $\bar{\Omega}$, the trajectory remains inside $\bar{\Omega}$ for all time.

Concerning the optimal visiting problem, note that the restriction to nonnegative $w_{j}$ is coherent with the model. Moreover, the set $\mathcal{B}$ may be viewed as a, sufficiently large, bounded set containing all the targets $\mathcal{T}_{j}$. The hypothesis (4.16) can be then artificially inserted in the model, just thinking that it would be reasonable to suppose that an optimal trajectory starts immediately pointing towards one of the targets, and hence not exit from a large, but bounded, set. However, using suitable penalization techniques in the study of the Hamilton-Jacobi equation, hypothesis (4.16) may be dropped.

The final cost of our Mayer problem is the function

$$
\Psi: \mathbb{R}^{n} \times \mathbb{R}^{m} \rightarrow\left[0,+\infty\left[, \quad(x, w) \mapsto w_{1}+w_{2}+\ldots+w_{m} .\right.\right.
$$


For every initial state $\left(x, w^{0}\right) \in \bar{\Omega}$, for every $t \geq 0$ and for every measurable control $\alpha \in \mathcal{A}$, we consider the cost

$$
J\left(x, w^{0}, t, \alpha\right):=\Psi\left(y_{\left(x, w^{0}\right)}(t ; \alpha), w_{\left(x, w^{0}\right)}(t ; \alpha)\right) .
$$

The Mayer problem is given by the minimization, over all measurable controls, of the cost $J$. Hence we define the value function $V: \bar{\Omega} \times[0,+\infty[\rightarrow[0,+\infty[$ as

$$
V\left(x, w^{0}, t\right):=\inf _{\alpha \in \mathcal{A}} J\left(x, w^{0}, t, \alpha\right) .
$$

We end this section by stating suitable estimates on the trajectories of (4.14).

Proposition 4.3. Let us suppose that (4.13),(4.15), (4.16) hold. For every $\tau>0$, there exists a constant $C_{\tau}$ such that $\forall \alpha \in \mathcal{A}, \forall t \in[0, \tau]$ and $\forall\left(x^{0}, w^{0}\right),\left(x^{1}, w^{1}\right) \in \bar{\Omega}$ the following holds

$$
\begin{gathered}
\left\|\left(y_{\left(x^{0}, w^{0}\right)}(t ; \alpha), w_{\left(x^{0}, w^{0}\right)}(t ; \alpha)\right)-\left(y_{\left(x^{1}, w^{1}\right)}(t ; \alpha), w_{\left(x^{1}, w^{1}\right)}(t ; \alpha)\right)\right\|_{\mathbb{R}^{n} \times \mathbb{R}^{m}} \\
\leq C_{\tau}\left(\left\|\left(x^{0}, w^{0}\right)-\left(x^{1}, w^{1}\right)\right\|_{\left.\mathbb{R}^{n} \times \mathbb{R}^{m}\right) .}\right.
\end{gathered}
$$

Proof. We write $y^{i}(\cdot)=y_{\left(x^{i}, w^{i}\right)}(\cdot ; \alpha)$ and $w^{i}(\cdot)=w_{\left(x^{i}, w^{i}\right)}(\cdot ; \alpha)$ for $i=0,1$. By standard estimates on trajectories we obtain the existence of $C_{1}>0$ such that

$$
\left\|y^{0}(\cdot)-y^{1}(\cdot)\right\|_{\mathcal{C}^{0}([0, \tau])} \leq C_{1}\left\|x^{0}-x^{1}\right\|_{\mathbb{R}^{n}} .
$$

Then, by Lipschitz continuity of Semiplay operator (3.8) and of the function $g_{j}$, we also get the existence of $C_{2}>0$ such that

$$
\left\|w^{0}(\cdot)-w^{1}(\cdot)\right\|_{\mathcal{C}^{0}([0, \tau])} \leq C_{2}\left\|\left(x^{0}, w^{0}\right)-\left(x^{1}, w^{1}\right)\right\|_{\mathbb{R}^{n} \times \mathbb{R}^{m}},
$$

from which we conclude.

\subsection{Continuity and DPP}

Theorem 4.4. Let us suppose that (4.13), (4.15) and (4.16) hold. Then the value function (4.17) is bounded and continuous in $\bar{\Omega} \times[0, T]$, for every $T>0$. 
Proof. Note that $\Psi$ is linear and hence uniformly continuous and bounded in $\bar{\Omega}$. The proof then standardly follows from the definition of $V$ and from Proposition 4.3.

Also the proof of the following crucial theorem follows from standard arguments, in particular recalling the semigroup property of the Semiplay operator (3.9).

Theorem 4.5 (DPP:Dynamic Programming Principle). Let us suppose that (4.13), (4.15), and (4.16) hold. Then, $\forall\left(x, w^{0}\right) \in \bar{\Omega}$, $\forall t>0$ and for every $\tau \in] 0, t]$, we have

$$
V\left(x, w^{0}, t\right)=\inf _{\alpha \in \mathcal{A}} V\left(y_{\left(x, w^{0}\right)}(\tau, \alpha), w_{\left(x, w^{0}\right)}(\tau ; \alpha), t-\tau\right) .
$$

\subsection{The discontinuous $\mathrm{H}-\mathrm{J}$ equation}

For every $(x, w) \in \bar{\Omega},(p, q) \in \mathbb{R}^{n} \times \mathbb{R}^{m}$, we define the following Hamiltonian:

$$
\begin{aligned}
& H(y, w, p, q)= \\
& \sup _{a \in A}\left\{-f(y, a) \cdot p+\sum_{j=1}^{m} q_{j} \chi\left(g_{j}(y), w_{j}\right)\left(D g_{j}(y) \cdot f(y, a)\right)^{-}\right\}
\end{aligned}
$$

where $\chi$ is the characteristic function of the bisector of first quadrant in the $\left(g_{j}, w_{j}\right)$-plane. Then $H$ is discontinuous in $\bar{\Omega} \times \mathbb{R}^{n} \times \mathbb{R}^{m}$. We consider the following Hamilton-Jacobi equation in $\bar{\Omega} \times] 0,+\infty[$

$$
u_{t}(x, w, t)+H\left(x, w, D_{x} u(x, w, t), D_{w} u(x, w, t)\right)=0,
$$

where $u_{t}$ indicate the derivative with respect to time, while $D_{x} u$ and $D_{w} u$ are the gradients of $u$ with respect to $y$ and $w$. We denote the lower semicontinuous and upper semicontinuous envelopes of $H$ respectively by $H_{*}$ and $H^{*}$. Simple calculations show that (recall that the positive and the negative part of $r \in \mathbb{R}$ are respectively $r^{+}=$ $\max (r, 0)$ and $\left.r^{-}=\max (-r, 0)\right)$ 


$$
\begin{aligned}
& H_{*}(x, w, p, q)= \\
& \sup _{a \in A}\left\{-f(x, a) \cdot p-\sum_{j=1}^{m} q_{j}^{-} \chi\left(g_{j}(x), w_{j}\right)\left(D g_{j}(x) \cdot f(x, a)\right)^{-}\right\}, \\
& H^{*}(x, w, p, q)= \\
& \sup _{a \in A}\left\{-f(x, a) \cdot p+\sum_{j=1}^{m} q_{j}^{+} \chi\left(g_{j}(x), w_{j}\right)\left(D g_{j}(x) \cdot f(x, a)\right)^{-}\right\} .
\end{aligned}
$$

Theorem 4.6. Let (4.13), (4.15), (4.16) hold. Then the value function $V$ is a continuous and bounded viscosity solution in $\bar{\Omega} \times] 0,+\infty[$ of the Hamilton-Jacobi equation (4.22), that is

$$
V_{t}(x, w, t)+H\left(x, w, D_{y} V(x, w, t), D_{w} V(x, w, t)\right)=0 .
$$

Proof. The continuity and the boundness come from Theorem 4.4; so it remains to prove that $V$ is a viscosity subsolution and supersoution of (4.23).

Take $\varphi \in \mathcal{C}^{1}(\bar{\Omega} \times] 0,+\infty[)$ such that $V-\varphi$ has a local maximum in $\left.\left(x, w^{0}, t\right) \in \bar{\Omega} \times\right] 0,+\infty\left[\right.$. Let us fix $\varepsilon>0$ and take $a_{\varepsilon} \in A$ such that

$$
\begin{aligned}
& -D_{y} \varphi\left(x, w^{0}, t\right) \cdot f\left(x, a_{\varepsilon}\right)-\sum_{j=1}^{m}\left(\frac{\partial \varphi}{\partial w_{j}}\left(x, w^{0}, t\right)\right)^{-} \chi\left(g_{j}(x),\left(w^{0}\right)_{j}\right) . \\
& \cdot\left(D g_{j}(x) \cdot f\left(x, a_{\varepsilon}\right)\right)^{-} \geq H_{*}\left(x, w^{0}, D_{x} \varphi\left(x, w^{0}, t\right), D_{w} \varphi\left(x, w^{0}, t\right)\right)+\varepsilon .
\end{aligned}
$$

We denote the trajectory starting from $\left(x, w^{0}\right)$ and corresponding to the constant control $\alpha \equiv a_{\varepsilon}$ simply by $(y(\cdot), w(\cdot))$. For $\tau>0$ sufficiently small, by the maximality of $\left(x, w_{0}, t\right)$, by DPP, and recalling that $\bar{\Omega}$ is invariant for the trajectories,, we get

$$
\begin{aligned}
& \varphi\left(x, w^{0}, t\right)-\varphi(y(\tau), w(\tau), t-\tau) \\
& \quad \leq V\left(x, w^{0}, t\right)-V(y(\tau), w(\tau), t-\tau) \leq 0 .
\end{aligned}
$$

Using the regularity of $\varphi$ and $g_{j}$ and recalling that $y$ and $w_{j}$ are absolutely continuous (in particular $y$ is even $C^{1}$ since the control is constant), and that $w_{j}$ satisfies (3.10) with $u$ replaced by $g_{j} \circ y$, we have 


$$
\begin{aligned}
& \varphi\left(x, w^{0}, t\right)-\varphi(y(\tau), w(\tau), t-\tau)= \\
& -\int_{0}^{\tau} \frac{d}{d s} \varphi(y(s), w(s), t-s) d s=-\left(\int _ { 0 } ^ { \tau } \left(D_{x} \varphi\left(x, w^{0}, t\right) \cdot f\left(x, a_{\varepsilon}\right)+\right.\right. \\
& -\sum_{j=1}^{m} \frac{\partial \varphi}{\partial w_{j}}\left(x, w^{0}, t\right) \chi\left(g_{j}(y(s)), w_{j}(s)\right)\left(D g_{j}(x) \cdot f\left(x, a_{\varepsilon}\right)\right)^{-}+ \\
& \left.-\varphi_{t}\left(x, w^{0}, t\right)\right) d s+o(\tau) \quad \text { for } \tau \rightarrow 0^{+}
\end{aligned}
$$

In order to handle the discontinuities in (4.25), just arguing on the signs of the involved factors, we get the following estimate:

$$
\begin{gathered}
\frac{\partial \varphi}{\partial w_{j}}\left(x, w^{0}, t\right) \chi\left(g_{j}(y(\tau)), w_{j}(\tau)\right)\left(D g_{j}(x) \cdot f\left(x, a_{\varepsilon}\right)\right)^{-} \\
\geq-\left(\frac{\partial \varphi}{\partial w_{j}}\left(x, w^{0}, t\right)\right)^{-} \chi\left(g_{j}(x), w_{j}^{0}\right)\left(D g_{j}(x) \cdot f\left(x, a_{\varepsilon}\right)\right)^{-} .
\end{gathered}
$$

Hence, by (4.24) and (4.25), we obtain

$$
\begin{aligned}
& \int_{0}^{\tau}-D_{y} \varphi\left(x, w^{0}, t\right) \cdot f\left(x, a_{\varepsilon}\right)-\sum_{j=1}^{m}\left(\frac{\partial \varphi}{\partial w_{j}}\left(x, w^{0}, t\right)\right)^{-} . \\
& \quad \cdot \chi\left(g_{j}(x), w_{j}^{0}\right)\left(D g_{j}(x) \cdot f\left(x, a_{\varepsilon}\right)\right)^{-} d s+ \\
& +\int_{0}^{\tau} \varphi_{t}\left(x, w^{0}, t\right) d s \leq o(\tau) .
\end{aligned}
$$

Now, since $H_{*}\left(x, w^{0}, D_{x} \varphi\left(x, w^{0}, t\right), D_{w} \varphi\left(x, w^{0}, t\right)\right)+\varepsilon$ is smaller than the argument of the first integral, then, dividing (4.26) by $\tau>0$ and letting $\tau \rightarrow 0^{+}$, we have

$$
H_{*}\left(x, w^{0}, D_{x} \varphi\left(x, w^{0}, t\right), D_{w} \varphi\left(x, w^{0}, t\right)\right)+\varphi_{t}\left(x, w^{0}, t\right) \leq-\varepsilon .
$$

So, as $\varepsilon \rightarrow 0^{+}$, we conclude that $V$ is a viscosity subsolution of (4.23).

To prove that $V$ is a viscosity supersolution of (4.23), we follow, as it is standard, in a similar way as the one above, considering a function $\varphi \in \mathcal{C}^{1}(\bar{\Omega} \times] 0,+\infty[)$ such that $V-\varphi$ has a local minimum in $\left.\left(x, w^{0}, t\right) \in \bar{\Omega} \times\right] 0,+\infty[$. Indeed, by DPP, for any $\varepsilon>0$ and $\tau>0$ sufficiently small, we find a measurable control $\bar{\alpha}$ such that, using the corresponding trajectory $(y(\cdot), w(\cdot))$, 


$$
\varphi\left(x, w^{0}, t\right)-\varphi(y(\tau), w(\tau), t-\tau) \geq-\varepsilon \tau
$$

Hence, we proceed similarly as before, using now the crucial estimate

$$
\begin{aligned}
& \frac{\partial \varphi}{\partial w_{j}}\left(x, w^{0}, t\right) \chi\left(g_{j}(y(\tau)), w_{j}(\tau)\right)\left(D g_{j}(x) \cdot f(x, \bar{\alpha}(\tau))\right)^{-} \\
& \leq\left(\frac{\partial \varphi}{\partial w_{j}}\left(x, w^{0}, t\right)\right)^{+} \chi\left(g_{j}(x), w_{j}^{0}\right)\left(D g_{j}(x) \cdot f(x, \bar{\alpha}(\tau))\right)^{-} .
\end{aligned}
$$

\section{$5 \quad$ Uniqueness}

In order to perform the classical analysis of uniqueness by a comparison result between sub- and supersolutions, the discontinuity of the Hamiltonian is a serious obstacle. Here, we are going to interpret such a discontinuity as a suitable boundary condition of Neumann-type, and then to study uniqueness for a continuous Hamilton-Jacobi equation with such Neumann conditions.

We define

$$
\Gamma:=\left\{(x, w) \in \bar{\Omega}: \exists j=1, \ldots, m \text { such that } w_{j}=g_{j}(x)>0\right\} \subseteq \partial \Omega,
$$

and note that $\Gamma$ is exactly the part of the boundary where the discontinuity of the $S P$-evolution representation (3.10) may play a role.

By Theorem 4.6 we know that $V$ is a continuous and bounded viscosity solution of (4.23) in $\bar{\Omega} \times] 0,+\infty$ [, with discontinuous Hamiltonian $H$ given by (4.21). Hence, since $\chi\left(g_{j}(x), w_{j}\right)=0$ out of $\Gamma$, we have that $V$ satisfies in the viscosity sense the following Hamilton-Jacobi equation

$$
\left.u_{t}(x, w, t)+H\left(x, w, D_{x} u(x, w, t), 0\right)=0, \text { in }(\bar{\Omega} \backslash \Gamma) \times\right] 0,+\infty[.
$$

Note that the Hamiltonian $(x, w, p, q) \mapsto H(x, w, p, 0)$ is now continuous. To equation (5.27) we must add the following boundary conditions of Neumann-type, in a viscosity sense as explained in section 3.3, 


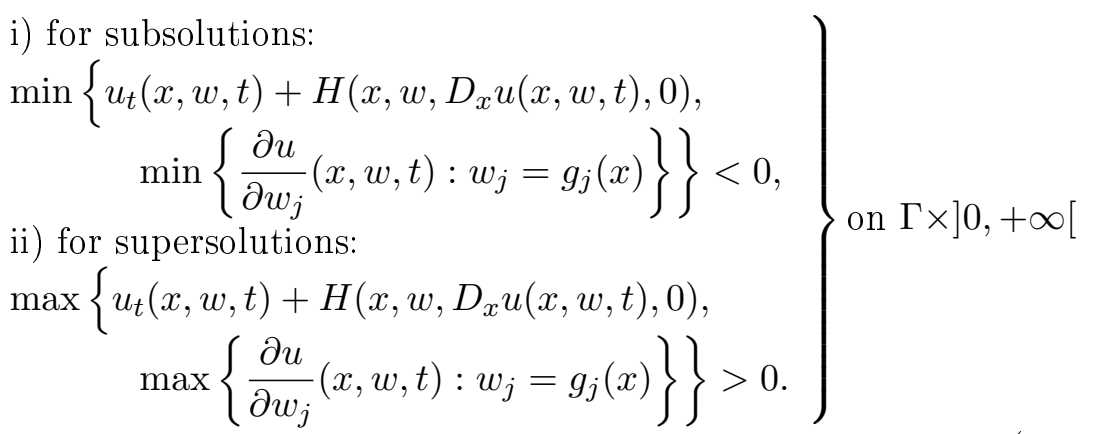

To understand the reasons of this conditions, let us take, for instance, a subsolution $u$ of $(4.23), \varphi \in C^{1}(\bar{\Omega} \times] 0,+\infty[)$, and $(x, w, t) \in$ $\Gamma \times] 0,+\infty[$ of maximum for $u-\varphi$ in $\bar{\Omega} \times] 0,+\infty[$. Hence there exists at least one $j \in\{1, \ldots, m\}$ such that $w_{j}=g_{j}(x)$. We assume that $\varphi_{t}(x, w, t)+H\left(x, w, D_{x} \varphi(x, w, t), 0\right)>0$. Recalling the lower semicontinuous envelope $H_{*}$, the following must hold: there exists $a \in A$ such that

$$
-\sum_{\substack{j=1 \\ w_{j}=g_{j}(x)}}^{m}\left(\frac{\partial \varphi}{\partial w_{j}}(x, w, t)\right)^{-}\left(D g_{j}(x) \cdot f(x, a)\right)^{-}<0,
$$

from which we deduce that at least one of those components of the gradient $D_{w} \varphi(x, w, t)$ is strictly negative, i.e:

$$
\min \left\{\frac{\partial \varphi}{\partial w_{j}}(x, w, t): w_{j}=g_{j}(x)\right\}<0 .
$$

We similarly obtain the other condition.

Theorem 5.1. Let as assume that (4.13), (4.15) and (4.16) hold. We consider $T \in] 0,+\infty[, u, v \in \mathcal{C}(\bar{\Omega} \times[0, T])$ bounded continuous functions such that they are respectively a viscosity subsolution and supersolution of (5.27), with boundary Neumann condition (5.28) in the viscosity sense. Then

$$
\sup _{\bar{\Omega} \times[0, T]}(u(x, w, t)-v(x, w, t)) \leq \sup _{\bar{\Omega}}(u(x, w, 0)-v(x, w, 0)) .
$$

Proof. First of all, note that, as it is standard, there exists a modulus of continuity $\omega$ such that, for all $(x, w),(y, z) \in \bar{\Omega}, p, q \in \mathbb{R}^{n}$, 


$$
\begin{aligned}
& |H(x, w, p, 0)-H(y, z, p, 0)| \leq \omega(\|x-y\|\|p\|), \\
& |H(x, w, p, 0)-H(x, w, q, 0)| \leq \omega(\|p-q\|) .
\end{aligned}
$$

Because of the geometry of $\bar{\Omega}$, there exists a positive definite symmetric $2 \times 2$ matrix $S$ such that, for every $(x, w) \in \Gamma$ and for every $j$ such that $w_{j}=g_{j}(x)$ (here $(\cdot)^{T}$ stays for the transpose, i.e. the column):

$$
S\left(\left(g_{j}(x), w_{j}\right)-\left(g_{j}\left(x^{*}\right), w_{j}^{*}\right)\right)^{T} \cdot(0,1) \geq 0, \quad \forall\left(x^{*}, w^{*}\right) \in \bar{\Omega} .
$$

Fixed $\varepsilon>0, \eta>0$, we define the following function in $(\bar{\Omega} \times[0, T])^{2}$

$$
\begin{aligned}
& \Phi((x, w, t),(y, z, s))=u(x, w, t)-v(y, z, s)-\frac{\|x-y\|^{2}+|t-s|^{2}}{2 \varepsilon}+ \\
& -\eta(t+s)-\sum_{j=1}^{m} \frac{S\left(g_{j}(x)-g_{j}(y), w_{j}-z_{j}\right)^{T} \cdot\left(g_{j}(x)-g_{j}(y), w_{j}-z_{j}\right)}{2 \varepsilon},
\end{aligned}
$$

Let $A:=\sup _{\bar{\Omega} \times\{0\}}(u-v)$ and we assume by contraddiction that there exist $\delta>0$ and $(\tilde{x}, \tilde{w}, \tilde{t}) \in \bar{\Omega} \times[0, T]$ such that

$$
(u-v)(\tilde{x}, \tilde{w}, \tilde{t})=A+\delta .
$$

We choose $\eta$ such that $2 \eta \tilde{t} \leq \frac{\delta}{2}$, then the following holds

$$
A+\frac{\delta}{2} \leq A+\delta-2 \eta \tilde{t}=\Phi((\tilde{x}, \tilde{w}, \tilde{t}),(\tilde{x}, \tilde{w}, \tilde{t})) \leq \sup _{(\bar{\Omega} \times[0, T])^{2}} \Phi .
$$

By the continuity of $\Phi$ in the compact $(\bar{\Omega} \times[0, T])^{2}$, there exist two points $(\bar{x}, \bar{w}, \bar{t}),(\bar{y}, \bar{z}, \bar{s}) \in \bar{\Omega} \times[0, T]$ such that

$$
\sup _{(\bar{\Omega} \times[0, T])^{2}} \Phi=\Phi((\bar{x}, \bar{w}, \bar{t}),(\bar{y}, \bar{z}, \bar{s})) .
$$

The following estimates are obtained by standard technique (see for instance Bardi-Capuzzo Dolcetta [3]), just suitably using our assumptions and in particular the positive definition of the matrix $S$

$$
\begin{aligned}
& \left|\bar{w}_{j}-\bar{z}_{j}\right| \leq \sqrt{C \varepsilon} \\
& \frac{\|\bar{x}-\bar{y}\|^{2}}{\varepsilon} \leq \frac{\|\bar{x}-\bar{y}\|^{2}+|\bar{t}-\bar{s}|^{2}}{\varepsilon} \leq \omega_{u+v}(\sqrt{\varepsilon}) \\
& \frac{\left|\bar{w}_{j}-\bar{z}_{j}\right|^{2}}{\varepsilon} \leq \omega_{u+v}(\sqrt{\varepsilon})
\end{aligned}
$$


where $C$ is a suitable constant and $\omega_{u+v}$ is the modulus of continuity in $\bar{\Omega}$ for $u+v$. We also get $\bar{t}, \bar{s}>0$.

We consider the following $\mathcal{C}^{1}$ functions:

$$
\begin{gathered}
\varphi(x, w, t)=v(\bar{y}, \bar{z}, \bar{s})+\frac{\|x-\bar{y}\|^{2}+|t-\bar{s}|^{2}}{2 \varepsilon}+\eta(t+\bar{s})+ \\
\sum_{j=1}^{m} \frac{S\left(g_{j}(x)-g_{j}(\bar{y}), w_{j}-\bar{z}_{j}\right)^{T} \cdot\left(g_{j}(x)-g_{j}(\bar{y}), w_{j}-\bar{z}_{j}\right)}{2 \varepsilon}, \\
\psi(y, z, s)=u(\bar{x}, \bar{w}, \bar{t})-\frac{\|\bar{x}-y\|^{2}+|\bar{t}-s|^{2}}{2 \varepsilon}-\eta(\bar{t}+s)+ \\
-\sum_{j=1}^{m} \frac{S\left(g_{j}(\bar{x})-g_{j}(y), \bar{w}_{j}-z_{j}\right)^{T} \cdot\left(g_{j}(\bar{x})-g_{j}(y), \bar{w}_{j}-z_{j}\right)}{2 \varepsilon} .
\end{gathered}
$$

Let us note that $u-\varphi$ has a maximum point in $(\bar{x}, \bar{w}, \bar{t})$, while $v-\psi$ has a minimum point in $(\bar{y}, \bar{z}, \bar{s})$. Then we distinguish two cases:

a) $(\bar{x}, \bar{w}, \bar{t}),(\bar{y}, \bar{z}, \bar{s}) \in(\bar{\Omega} \backslash \Gamma) \times] 0, T]$,

b) $(\bar{x}, \bar{w}, \bar{t}) \in \Gamma \times] 0, T]$ or $(\bar{y}, \bar{z}, \bar{s}) \in \Gamma \times] 0, T]$.

a) Since $u$ and $v$ are respectively a viscosity sub- and supersolution of (5.1), also recalling Lemma 3.5 holds, the following are satisfied

$$
\begin{gathered}
\varphi_{t}(\bar{x}, \bar{w}, \bar{t})+H\left(\bar{x}, \bar{w}, D_{x} \varphi(\bar{x}, \bar{w}, \bar{t}), 0\right) \leq 0 \\
\psi_{t}(\bar{y}, \bar{z}, \bar{s})+H\left(\bar{y}, \bar{z}, D_{x} \psi(\bar{y}, \bar{z}, \bar{s}), 0\right) \geq 0 .
\end{gathered}
$$

By just a calculation this means

$$
2 \eta \leq H\left(\bar{y}, \bar{z}, D_{y} \psi(\bar{y}, \bar{z}, \bar{s}), 0\right)-H\left(\bar{x}, \bar{w}, D_{x} \varphi(\bar{x}, \bar{w}, \bar{t}), 0\right)
$$

Then, using (5.29), the Lipschitz continuity of $g_{j}$ and $D g_{j}$ in $\overline{\mathcal{B}}$, the estimates (5.33), we get

$$
\left|H\left(\bar{y}, \bar{z}, D_{y} \psi(\bar{y}, \bar{z}, \bar{s}), 0\right)-H\left(\bar{x}, \bar{w}, D_{x} \varphi(\bar{x}, \bar{w}, \bar{t}), 0\right)\right| \rightarrow 0 \quad \varepsilon \rightarrow 0^{+} .
$$

So, reconsidering (5.34), we find that $2 \eta \leq 0$ which is a contradiction. b) If $(\bar{x}, \bar{w}, \bar{t}) \in \Gamma \times] 0, T]$ or $(\bar{y}, \bar{z}, \bar{s}) \in \Gamma \times] 0, T]$ hold, then, by (5.30), for every $j$ such that $\bar{w}_{j}=g_{j}(\bar{x})$ or $\bar{z}_{j}=g_{j}(\bar{y})$, the following are respectively satisfied

$$
\begin{gathered}
S\left(\frac{\left(g_{j}(\bar{x}), \bar{w}_{j}\right)-\left(g_{j}(\bar{y}), \bar{z}_{j}\right)}{2 \varepsilon}\right)^{T} \cdot(0,1) \geq 0 \\
S\left(\frac{\left(g_{j}(\bar{x}), \bar{w}_{j}\right)-\left(g_{j}(\bar{y}), \bar{z}_{j}\right)}{2 \varepsilon}\right)^{T} \cdot(0,-1) \leq 0,
\end{gathered}
$$


which, by a direct calculation, respectively means

$$
\frac{\partial \varphi}{\partial w_{j}}(\bar{x}, \bar{w}, \bar{t}) \geq 0 \quad \frac{\partial \psi}{\partial w_{j}}(\bar{y}, \bar{z}, \bar{s}) \leq 0,
$$

from which, by the Neumann conditions (3.3), we obtain

$$
\begin{gathered}
\varphi_{t}(\bar{x}, \bar{w}, \bar{t})+H\left(\bar{x}, \bar{w}, D_{x} \varphi(\bar{x}, \bar{w}, \bar{t}), 0\right) \leq 0 \\
\psi_{t}(\bar{y}, \bar{z}, \bar{s})+H\left(\bar{y}, \bar{z}, D_{x} \psi(\bar{y}, \bar{z}, \bar{s}), 0\right) \geq 0
\end{gathered}
$$

and we conclude as in the previous case.

Let us now note that the value function $V$ (4.17) obviously satisfies the initial condition

$$
V(x, w, 0)=\Psi(x, w) \quad \forall(x, w) \in \bar{\Omega} .
$$

Hence, as it is standard, from Theorem 5.1, and from our interpretation of the discontinuity of the Hamiltonian as Neumann-type boundary conditions, we obtain the following uniqueness result.

Theorem 5.2. With the same assumptions of Theorem 5.1, the value function $V$ is the unique continuous and bounded viscosity solution of the following discontinuous Cauchy problem

$$
\left\{\begin{array}{l}
\left.V_{t}+H\left(x, w, D_{x} V, D_{w} V\right)=0 \text { in } \bar{\Omega} \times\right] 0,+\infty[ \\
V(x, w, 0)=\Psi(x, w)
\end{array}\right.
$$

\section{Remarks and extensions}

Remark 6.1. The viscosity solution approach to the state constraints problems was first studied by Soner [13]. It concerns the case where the trajectory is constrained to remain inside a closed set, and, in our setting, this consists of the constraint $y_{x}(t) \notin \stackrel{\circ}{\mathcal{T}_{j}}$ for every $j=1, \ldots, m$ (corresponding for instance to the physical interpretation of the targets as planets). Inserting such a constraint in our optimal visiting problem, and following Soner [13], we would get an Hamilton-Jacobi equation satisfied in a subsolution-way outside of the sets $\stackrel{\circ}{\mathcal{T}}_{j}$, and in a supersolution-way till to the boundary of $\stackrel{\circ}{\mathcal{T}}_{j}, j=1, \ldots, m$. Unfortunately when, in order to get the desired comparison result, we try to perform the suitable penalization technique as in [13], in particular the 
technique for keeping the maxima inside the interior of the set, some difficulties seem to arise for handling both that penalization technique from one side and the variables $w_{j}$ with the Neumann conditions from the other side. However, we did not check the details.

Remark 6.2 Another possible way for formalizing the minimal time visiting problem may probably be by an infinite horizon problem, still subject to the controlled evolution (4.14). In such a case, the objective would be the minimization of the cost

$$
J_{\lambda}(x, w, \alpha)=\int_{0}^{+\infty} e^{-\lambda t}\left(w_{1}(t)+\ldots+w_{m}(t)\right) d t,
$$

for a suitable $\lambda>0$ This choice is guided by the idea that, to minimize $J_{\lambda}$, probably it is convenient to send to zero all the components $w_{j}$ as soon as possible, and this means visiting the target sets. Actually, it may happen that the minimal cost is achieved with $0<w_{j}<\varepsilon$, for some $\varepsilon>0$, so the targets are not visited, but we certainly would have passed close enough to them.

For any fixed discount factor $\lambda>0$ we still perform a similar analysis as for the Mayer problem, and we characterize the value function $V_{\lambda}$ as the unique viscosity solution of a discontinuous Hamilton-Jacobi equation (see Benetton [5] for details).

An interesting issue to be studied would be the related ergodic problem, that is the investigation of the behavior of the sequence of functions $\lambda V_{\lambda}:=\inf _{\alpha}\left\{\lambda J_{\lambda}(x, w, \alpha)\right\}$ for $\lambda \rightarrow 0$. Such an analysis may lead to characterize the possible limit $V$ as the solution of a suitable limit Hamilton-Jacobi equation, and to recognize the limit $V$ exactly as our optimal visiting function.

\section{References}

[1] F. Bagagiolo, Dynamic programming for some optimal control problems with hysteresis, NoDEA 9 (2002),149-174.

[2] F. Bagagiolo, Viscosity solutions for an optimal control problem with Preisach hysteresis nonlinearities, ESAIM: Control, Optimisation, and Calculus of Variations, 10, (2004), 271-294.

[3] M. Bardi, I. Capuzzo Dolcetta, Optimal Control and Viscosity Solution of Hamilton-Jacobi-Bellman Equation, Birkhäuser, Boston, 1997. 
[4] R. Bellman, Dynamic programming treatment of the travelling salesman problem, Journal of ACM, 9 (1962), 61-63 .

[5] M. Benetton, Su di un problema di visita ottima, Master Thesis in Mathematics, University of Trento, July 2010.

[6] M.G. Crandall, P.L. Lions, Viscosity solutions of Hamilton-Jacobi equations, Trans. Amer. Math. Soc. 277 (1983), 1-42.

[7] M.G. Crandall, L.C. Evans, P.L. Lions, Some properties of viscosity solutions of Hamilton-Jacobi equations, Trans. Amer. Math. Soc. 282 (1984), 487-502.

[8] M.V. Day, Neumann-type boundary conditions for Hamilton-Jacobi equations in smooth domains, Appl. Math. Optim., 53 (2006), 359381.

[9] A. Gudovich, M. Quincampoix, Optimal control with hysteresis nonlinearity and multidimensional play operator, Preprint 2009.

[10] A. Ilchmann, H. Logemann, E. P. Ryan, Tracking with prescribed transient performance for hysteretic systems, SIAM J. Control Optim., 48 (2010), 4731-4752.

[11] H. Ishii, A boundary value problem of the Dirichlet type for the Hamilton-Jacobi equations, Ann. Scuola Norm. Pisa Cl. Sci. 16 (1989), 105-135.

[12] P.L. Lions, Neumann type boundary conditions for HamiltonJacobi equations, Duke Math. J. 52 (1985), 793-820.

[13] H.M. Soner, Optimal control with state-space constraint, SIAM J. Control Optim., 24 (1983),552-561.

[14] A. Visintin, Differential Models of Hysteresis, Springer, Heildeberg, 1994. 